ARTICLE

https://doi.org/10.1038/s41467-019-09269-9

\title{
Boundary activated hydrogen evolution reaction on monolayer $\mathrm{MoS}_{2}$
}

Jianqi Zhu ${ }^{1,2}$, Zhi-Chang Wang ${ }^{3}$, Huijia Dai ${ }^{4}$, Qinqin Wang ${ }^{1,5}$, Rong Yang ${ }^{1,5}$, Hua Yu ${ }^{1}$, Mengzhou Liao ${ }^{1,5}$, Jing Zhang ${ }^{1}$, Wei Chen ${ }^{1}$, Zheng Wei ${ }^{1,5}, \mathrm{Na} \mathrm{Li}^{1,5}$, Luojun Du ${ }^{1}$, Dongxia Shi ${ }^{1,5}$, Wenlong Wang ${ }^{1,5}$, Lixin Zhang ${ }^{4}$, Ying Jiang (1) ${ }^{3,6,7}$ \& Guangyu Zhang $1,5,6,8$

Recently, monolayer molybdenum disulphide $\left(\mathrm{MoS}_{2}\right)$ has emerged as a promising and non-precious electrocatalyst for hydrogen evolution reaction. However, its performance is largely limited by the low density and poor reactivity of active sites within its basal plane. Here, we report that domain boundaries in the basal plane of monolayer $\mathrm{MoS}_{2}$ can greatly enhance its hydrogen evolution reaction performance by serving as active sites. Two types of effective domain boundaries, the $2 \mathrm{H}-2 \mathrm{H}$ domain boundaries and the $2 \mathrm{H}-1 \mathrm{~T}$ phase boundaries, were investigated. Superior hydrogen evolution reaction catalytic activity, long-term stability and universality in both acidic and alkaline conditions were achieved based on a multihierarchy design of these two types of domain boundaries. We further demonstrate that such superior catalysts are feasible at a large scale by applying this multi-hierarchy design of domain boundaries to wafer-scale monolayer $\mathrm{MoS}_{2}$ films.

\footnotetext{
${ }^{1}$ CAS Key Laboratory of Nanoscale Physics and Devices, Institute of Physics, Chinese Academy of Sciences, Beijing 100190, China. ${ }^{2}$ School of Physics and Electronic Engineering, Sichuan Normal University, Chengdu, Sichuan 610101, China. ${ }^{3}$ International Center for Quantum Materials, School of Physics, Peking University, Beijing 100871, China. ${ }^{4}$ School of Physics, Nankai University, Tianjin 300071, China. ${ }^{5}$ School of Physical Sciences, University of Chinese Academy of Sciences, Beijing 100190, China. ${ }^{6}$ Collaborative Innovation Center of Quantum Matter, Beijing 100190, China. ${ }^{7}$ CAS Center for Excellence in Topological Quantum Computation, University of Chinese Academy of Sciences, Beijing 100190, PR China. ${ }^{8}$ Beijing Key Laboratory for Nanomaterials and Nanodevices, Beijing 100190, China. These authors contributed equally: Jianqi Zhu, Zhi-Chang Wang, Huijia Dai. Correspondence and requests for materials should be addressed to R.Y. (email: ryang@iphy.ac.cn) or to L.Z. (email: Ixzhang@nankai.edu.cn) or to Y.J. (email: yjiang@pku.edu.cn) or to
}

G.Z. (email: gyzhang@iphy.ac.cn) 
ydrogen evolution reaction (HER) process is crucial to the production of hydrogen, the most efficient and environmental-friendly fuel. Platinum $(\mathrm{Pt})$ and $\mathrm{Pt}$-based materials are known as the best electrocatalysts for HER so far, but they are also very scarce and expensive ${ }^{1}$. Recently, molybdenum disulfide $\left(\mathrm{MoS}_{2}\right)$ has emerged as an active, earth-abundant, and inexpensive alternative to $\mathrm{Pt}$ and Pt-based electrocatalysts $^{2-7}$. It is generally believed that the catalytic activity of $\mathrm{MoS}_{2}$ originates from its edges while its basal plane is rather inert, which limits the practical application of this material for $\mathrm{HER}^{8-11}$. In order to overcome the limited catalytic activity of the $\mathrm{MoS}_{2}$ basal plane, various techniques have been developed, such as phase engineering ${ }^{12-15}$, interface electronic coupling ${ }^{16}$, introducing active unsaturated defects ${ }^{14}$ and strain ${ }^{17}$. These techniques could improve the restricted factors (poor conductivity and limited active sites) for the potential of $\mathrm{MoS}_{2}$ in HER $^{18-20}$. Recently, a pioneer strategy has been proposed by introducing $S$ vacancies into the basal plane ${ }^{17,21-25}$, where gap states around the Fermi level allow hydrogen to bind directly to exposed Mo atoms. Considering the presence of dangling bonds in vacancy defects, these vacancy defects in $\mathrm{MoS}_{2}$ are easy to be poisoned and would lead to the surface instability from a HER point of view. To fully exploit $\mathrm{MoS}_{2}$ materials in realistic application, searching for alter

Herein, we report a facile route toward the activation of the monolayer $\mathrm{MoS}_{2}$ basal plane for HER by introducing domain boundaries, including both $2 \mathrm{H}-2 \mathrm{H}$ domain boundaries and $2 \mathrm{H}-1 \mathrm{~T}$-phase boundaries. We found that the domain boundaries can provide ultrahigh-density active sites, while still maintain the surface stability. Utilizing a multi-hierarchy design of these two types of boundaries, we are able to achieve a high basal-plane electrocatalytic performance with an exchange current density of $0.57 \times 10^{-4} \mathrm{~A} \mathrm{~cm}^{-2}$, a Tafel slope of $73 \mathrm{mV} \mathrm{dec}^{-1}$, and a remarkable long-term operation stability over $200 \mathrm{~h}$. We also demonstrate that such catalysts are scalable, e.g., over 4 -inch wafer scale, pushing a crucial technological step toward practical applications.

\section{Results}

2H-2H domain boundaries and 2H-1T-phase boundaries for HER. In this study, we investigated both single-crystalline and polycrystalline $2 \mathrm{H}$-phase monolayer $\mathrm{MoS}_{2}$ samples grown by chemical vapor deposition (CVD) for HER. The former samples (type-I) were grown on sapphire substrates with individual domain size of a few hundred micrometers (refer to ref. ${ }^{25}$ for our previous work $)^{26}$. The latter samples were continuous film samples grown either on sapphire substrates (type-II) with highly orientated domains of a few micrometers (refer to ref. ${ }^{26}$ for our previous work $)^{27}$ or on $\mathrm{SiO}_{2} / \mathrm{Si}$ substrates (type-III) with randomly orientated domains of a few hundred nanometers (refer to ref. ${ }^{27}$ for our previous work) ${ }^{28}$. Figure 1a-c show typical optical and false-color transmission electron microscope (TEM) images of these different monolayer $\mathrm{MoS}_{2}$ samples to illustrate their domain sizes and the $2 \mathrm{H}-2 \mathrm{H}$ domain boundaries. Hence, we have three types of $\mathrm{MoS}_{2}$ samples for comparable investigation of the HER performances: type-I free of $2 \mathrm{H}-2 \mathrm{H}$ domain boundaries, type-II with low density of $2 \mathrm{H}-2 \mathrm{H}$ domain boundaries, and typeIII with high density of $2 \mathrm{H}-2 \mathrm{H}$ domain boundaries (further details in Supplementary Fig. 1). Note that the $2 \mathrm{H}-2 \mathrm{H}$ domain boundaries in our $\mathrm{MoS}_{2}$ samples are not perfectly straight. These boundaries usually consist of various configurations, typically including arrays of 4-6 rings (4|6), 6-8 rings (6|8), 5-7 rings (5| 7 ), and 4-4 rings (4|4) as shown in Supplementary Fig. 2. The high-resolution TEM and X-ray photoelectron spectroscopy (XPS) were used to confirm that all samples are of high quality with low-defect density and very clean surfaces even after transfer processes (Supplementary Fig. 3). Hence, the influence of defects in different types of samples can be excluded.

In order to investigate whether the $2 \mathrm{H}-2 \mathrm{H}$ domain boundaries could serve as active sites in HER, local probe characterizations were first performed (further details in Supplementary Note 1 and Supplementary Fig. 4). Figure $1 \mathrm{~d}$ and e show two typical HER devices fabricated from type-I and type-III samples, respectively. Note that these devices were protected by polymethyl methacrylate (PMMA) masks with only small windows exposed on the $\mathrm{MoS}_{2}$ samples in the center to avoid the contribution from the $\mathrm{MoS}_{2}$ edges during the HER characterizations. The corresponding HER polarization curves and Tafel plots of both devices are shown in Fig. 1f, g, respectively. It can be clearly seen that type-III samples have better catalytic performances than type-I samples as evidenced by the drop of the overpotential from $\sim 375 \mathrm{mV}$ to $\sim 325 \mathrm{mV}$ at current density of $10 \mathrm{~A} \mathrm{~cm}^{-2}$ and the Tafel slope from $\sim 110$ to $\sim 95 \mathrm{mV} \mathrm{dec}-1$. Since the qualities of the two samples are very similar, except for the density of domain boundaries, we thus can draw a conclusion that the enhanced HER activity in typeIII samples comes from the $2 \mathrm{H}-2 \mathrm{H}$ domain boundaries.

Then we explored the possibility of further introducing $2 \mathrm{H}-1 \mathrm{~T}$ phase boundaries as active sites in HER. In order to produce such boundaries, we performed post low-energy Ar-plasma bombardments on pristine type-I and type-III samples to induce the $2 \mathrm{H}$-to1T-phase transition (refer to ref. ${ }^{28}$ for our previous work) ${ }^{29}$. Note that this phase transition is quite local and the resulted samples consist of a mosaic texture of nearly half $2 \mathrm{H}$ - and half $1 \mathrm{~T}$-phases, exhibiting an average domain size of a few nanometers and highdensity phase boundaries (Supplementary Fig. 5). HER performance of two typical heterophase devices similar to those described above is shown in Fig. 1f, g. For type-I sample after phase transition, a drop of the overpotential from $\sim 370$ to $\sim 260 \mathrm{mV}$ at current density of $10 \mathrm{~A} \mathrm{~cm}^{-2}$ and the Tafel slope from $\sim 110$ to $\sim 85 \mathrm{mV}$ $\mathrm{dec}^{-1}$ can be clearly seen, suggesting that $2 \mathrm{H}-1 \mathrm{~T}$-phase boundaries are more efficient for HER as active reaction sites than $2 \mathrm{H}-2 \mathrm{H}$ boundaries. Besides, the metallic $1 \mathrm{~T}$ phase offers better charge transport capability than $2 \mathrm{H}$ phase, which is consistent with previous report ${ }^{16}$. Notably, the heterophase type-III sample exhibits the lowest overpotential of $200 \mathrm{mV}$ and Tafel slope of

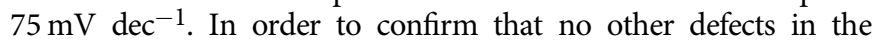
heterophase structure contributed prominently to the enhanced HER activity, we performed atomic force microscopy (AFM) and STM characterizations. Only few S vacancies $(\sim 1.9 \%)$ having little effect on HER can be found without any other defects in the heterophase sample (details in Supplementary Figs. 6-7 and Supplementary Note 2) ${ }^{17,25,30}$. Particularly, we also found that these $S$ vacancies would not introduce gap states that allow favorable hydrogen adsorption (Supplementary Fig. 7). We have also performed thermal annealing for our phase-changed samples to investigate the effect of phase boundaries. Thermal annealing was carried out in vacuum at $600^{\circ} \mathrm{C}$ for $1 \mathrm{~h}$. This process can recover from all $1 \mathrm{~T}$ phases back to $2 \mathrm{H}$ phases in our sample (as confirmed in Supplementary Fig. 8a). Meanwhile, after annealing treatment, the HER performance degrades to the same level of pristine $\mathrm{MoS}_{2}$, as shown in Supplementary Fig. 8b. On the basis of these results, we concluded that the phase boundaries are dominant active sites in our heterophase $\mathrm{MoS}_{2}$ samples while those $S$ vacancies contribute less to enhance the HER performance. Thus the composite structure containing both high-density domain and phase boundaries is the most promising candidate for HER.

Mechanism of the activation of the $\mathrm{MoS}_{2}$ basal plane by boundaries. In order to confirm the role of $2 \mathrm{H}-1 \mathrm{~T}$-phase boundaries in the basal plane of $\mathrm{MoS}_{2}$ in HER, we performed 
a
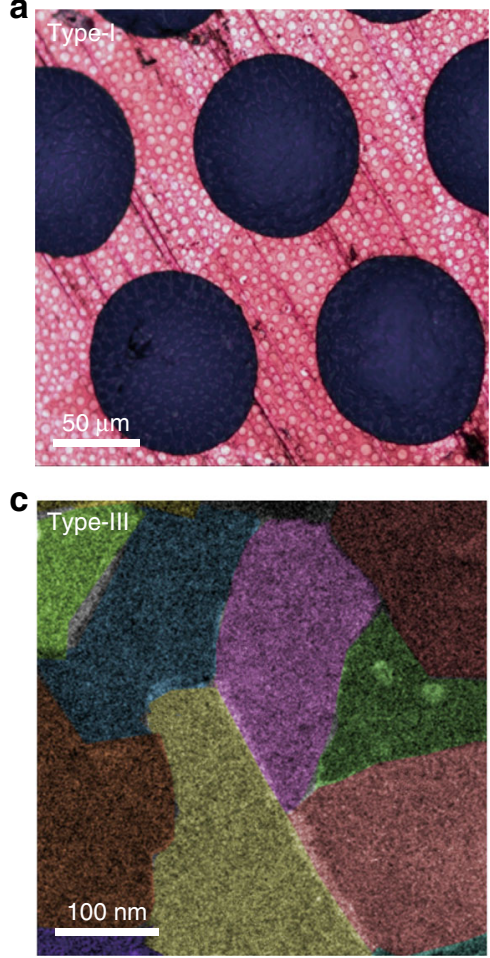

$f$

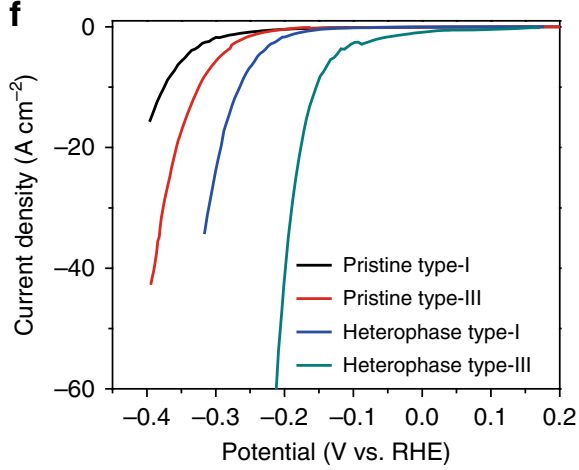

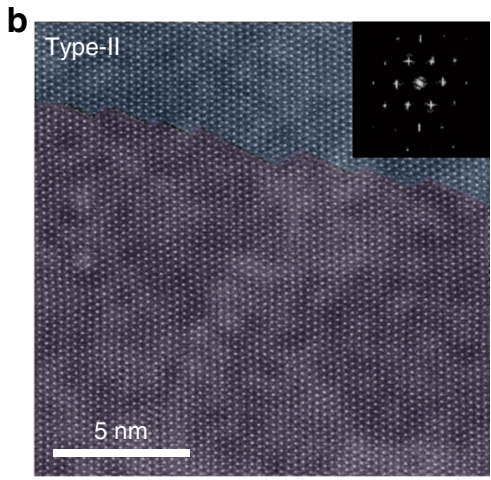

d
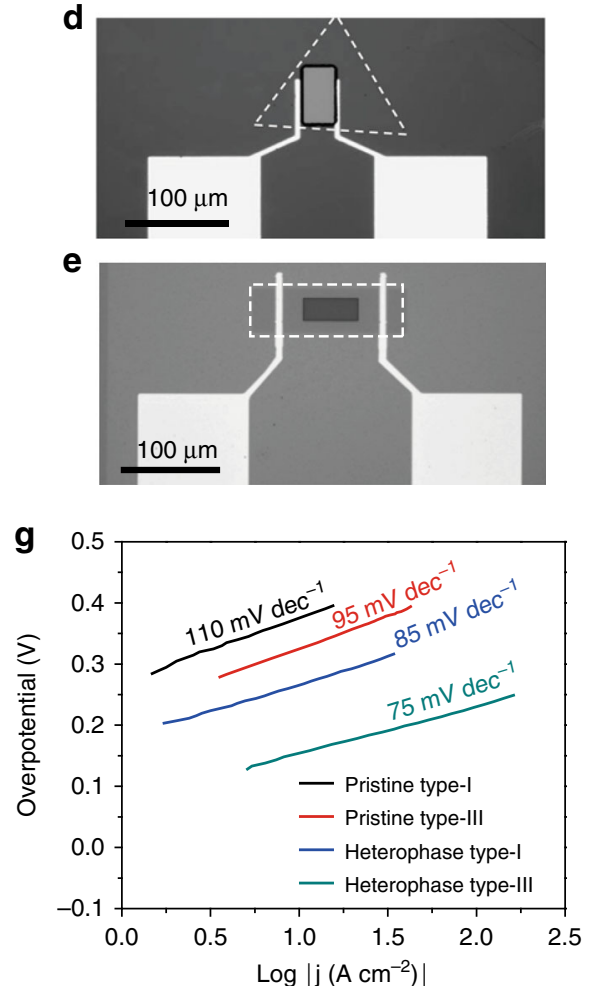

Fig. 1 Activity of $2 \mathrm{H}-2 \mathrm{H}$ domain boundaries and $2 \mathrm{H}-1 \mathrm{~T}$-phase boundaries for HER. a Optical image of the type-I MoS 2 with individual domain size of few

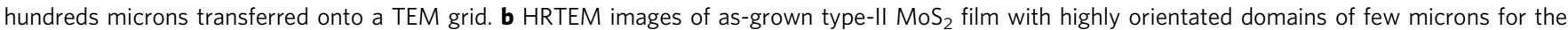
domain sizes. c False-color dark-field TEM image of type-III $\mathrm{MoS}_{2}$ with high-density $2 \mathrm{H}-2 \mathrm{H}$ domain boundaries. d, e Optical microscope image of a individual $\mathrm{MoS}_{2}$ single-crystal domain without $2 \mathrm{H}-2 \mathrm{H}$ domain boundaries (d) and polycrystalline $\mathrm{ML}_{-}-\mathrm{MoS}_{2}$ with domain boundaries (e). Dashed regions indicate the HER window opened on the basal plane. $\mathbf{f}$ Polarization curves of the pristine type-I MoS $\mathrm{S}_{2}$ (without any domain boundaries), pristine type-III $\mathrm{MoS}_{2}$ (with $2 \mathrm{H}-2 \mathrm{H}$ domain boundaries), heterophase type-I MoS (with $2 \mathrm{H}-1 \mathrm{~T}$-phase domain boundaries), and heterophase type-III MoS (with both $2 \mathrm{H}-2 \mathrm{H}$ and $2 \mathrm{H}-1 \mathrm{~T}$ domain boundaries), respectively. $\mathbf{g}$ Tafel plots of the corresponding curves in $\mathbf{f}$

scanning tunneling microscopy (STM) and scanning tunneling spectroscopy (STS) characterizations. Since STM/STS measurements require conductive substrates, $\mathrm{MoS}_{2}$ samples were epitaxially grown on graphite by CVD and treated by Ar-plasma to induce phase transitions subsequently. Figure $2 \mathrm{a}$ shows a typical topographic STM image of the monolayer heterophase $\mathrm{MoS}_{2}$, where bright and dark regions correspond to the $2 \mathrm{H}$ and $1 \mathrm{~T}$ phases, respectively. A zoom-in image at one-phase boundary is shown in Fig. $2 \mathrm{~b}$ with the top sulfur (S) atoms clearly resolved. The sliding of $\mathrm{S}$ atoms in $1 \mathrm{~T}$ phase with respect to the $2 \mathrm{H}$ phase further confirmed coexistence of the two phases (details in Supplementary Fig. 9). As shown in Fig. 2c, 2H-phase domains have bandgaps as usual, e.g., $2.5 \mathrm{eV}$; in contrast, $1 \mathrm{~T}$-phase domains are metallic without bandgaps.

In HER process, stable hydrogen adsorption at active sites is a crucial step. Therefore, we simulated this step via hydrogenating the heterophase sample surface by the atomic hydrogen (see Supporting Information for more details). After hydrogenation, additional depression features with atomic-size width appear exactly at the phase boundaries (Fig. 2d, e), suggesting that the atomic hydrogen prefers to adsorb at the $S$ sites of phase boundary. Density functional theory (DFT) calculations were also carried out to confirm the STM observations. Simulated results indicate that the formation of $\mathrm{S}-\mathrm{H}$ covalent bonds can lead to a significant reduction of the density of states (DOS) of $\mathrm{S}$ atoms around the Fermi level (Fig. 2f), which is consistent with the apparent depression observed in the STM images due to the adsorption of $\mathrm{H}$ atoms on the $\mathrm{S}$ atoms.

Next, we performed further DFT calculations on the $2 \mathrm{H}-2 \mathrm{H}$ grain boundaries and the $2 \mathrm{H}-1 \mathrm{~T}$-phase boundaries to investigate their catalytic activities (see Supporting Information for computational details). Figure $3 \mathrm{a}, \mathrm{b}$ show the structural model of 


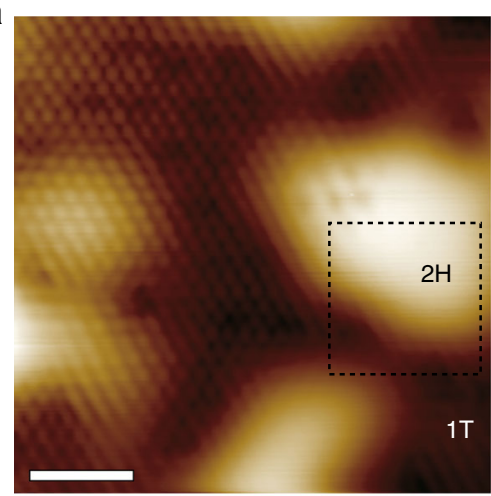

d

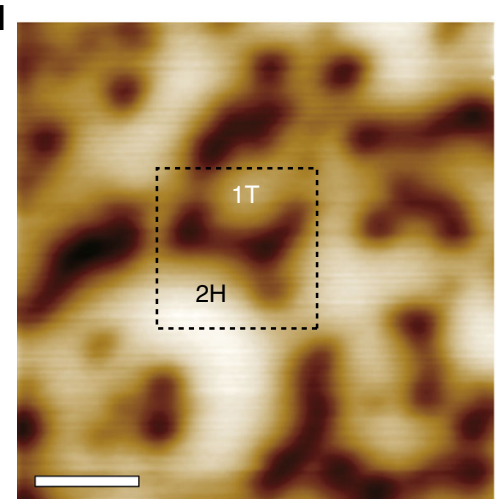

b

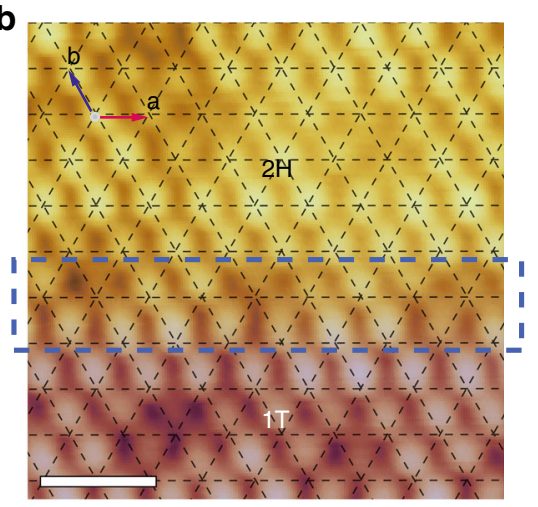

e

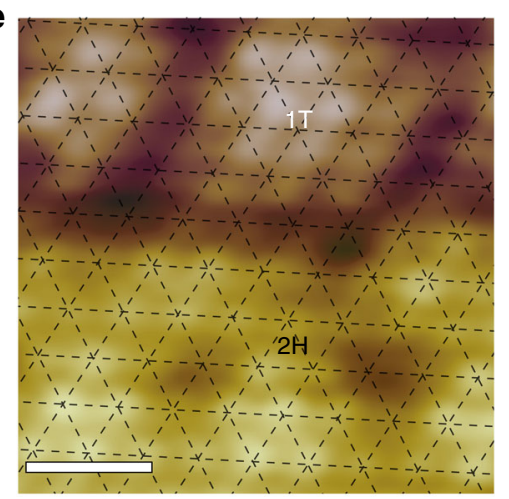

C

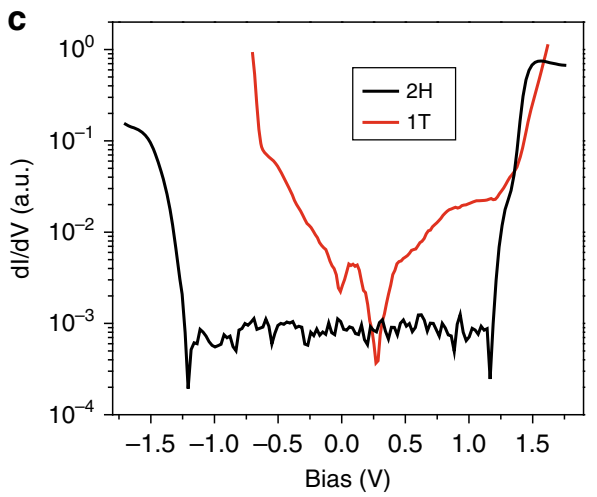

f

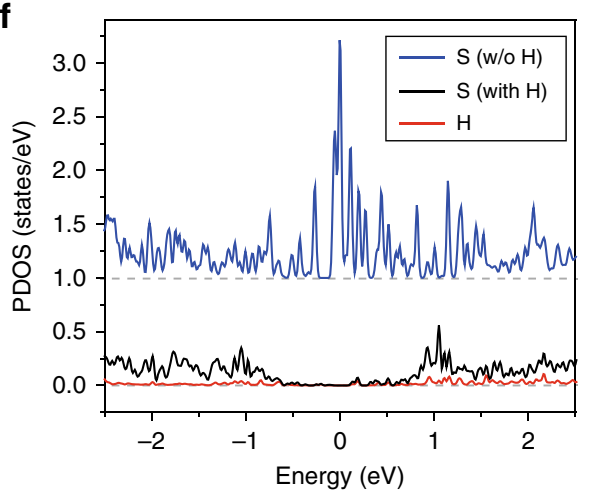

Fig. 2 Hydrogen adsorption at 2H-1T-phase boundaries. a STM topography of as-treated $\mathrm{MoS}_{2}$ showing mixed $2 \mathrm{H}$ (bright) and $1 \mathrm{~T}$ (dark) domains b Zoom-in image of a domain boundary as denoted by a dashed square in $\mathbf{a}$, where $2 \mathrm{H}$ and $1 \mathrm{~T}$ phases are rendered with yellow and purple colors, respectively. The lattice grid of $2 \mathrm{H}$ phase is superimposed on the image to highlight the lateral sliding of $\mathrm{S}$ atoms in $1 \mathrm{~T}$ phase. The black arrows indicate the sliding direction [120]. The two in-plane primitive vectors are shown in the upper left of $\mathbf{b}$. c dl/dV spectra taken on the $2 \mathrm{H}$ and $1 \mathrm{~T}$ phases, suggesting the semiconducting $2 \mathrm{H}$ phase with a $2.5-\mathrm{eV}$ bandgap and metallic $1 \mathrm{~T}$ phase. d STM image of the $\mathrm{MoS}_{2}$ after hydrogenation, showing prominent depression features. The sample bias was chosen such that the apparent heights of $2 \mathrm{H}$ and $1 \mathrm{~T}$ phases become similar, to highlight the depression features. $\mathbf{e}$ Zoom-in image of the depression features as denoted by a dashed square in $\mathbf{d}$, indicating that the depression features are located exactly at the boundary of $2 \mathrm{H}$ and $1 T$ phases. Set points of the STM images: a: $1.5 \mathrm{~V}, 50 \mathrm{pA} ; \mathbf{b}: 1 \mathrm{~V}, 10 \mathrm{pA} ; \mathbf{d}: 2 \mathrm{~V}, 10 \mathrm{pA}$; e: $0.5 \mathrm{~V}, 50 \mathrm{pA}$. Set point of dl/dV spectra:1.5 V, 50 pA c. f Projected density of states (PDOS) of $\mathrm{S}$ and $\mathrm{H}$ atoms at the phase boundary before and after hydrogenation. Blue curve: PDOS of $\mathrm{S}$ atoms before hydrogenation, black curve: PDOS of $\mathrm{S}$ atoms with $\mathrm{H}$ bonded, red curve: PDOS of absorbed $\mathrm{H}$ atoms. Scale bar: $2 \mathrm{~nm} \mathbf{a}, 0.7 \mathrm{~nm} \mathbf{b}, 1.5 \mathrm{~nm} \mathbf{d}, 0.7 \mathrm{~nm} \mathbf{e}$

$2 \mathrm{H}-1 \mathrm{~T}-$ phase boundaries and four typical kinds of $2 \mathrm{H}-2 \mathrm{H}$ boundary configurations $(4|8,6| 8,4|4,5| 7)^{24}$. Figure $3 \mathrm{c}$ shows the calculated Gibbs free energy of the adsorbed atomic hydrogen $\left(\Delta \mathrm{G}_{\mathrm{H}^{*}}\right)$. Note that $\Delta \mathrm{G}_{\mathrm{H}^{*}}$ is a widely accepted indicator for the catalytic activity and the optimal value is $\Delta \mathrm{G}_{\mathrm{H}^{*}}=0 \mathrm{eV}$, where hydrogen is bounded neither too strongly nor too weakly ${ }^{31}$. For comparison, we also performed calculations on Pt (111) surface, basal plane of $2 \mathrm{H}-\mathrm{MoS}_{2}$ and $1 \mathrm{~T}-\mathrm{MoS}_{2}$ with the same sized supercells as for the phase boundaries, yielding $\Delta \mathrm{G}_{\mathrm{H}^{*}}=-0.18 \mathrm{eV}$, $1.87 \mathrm{eV}$, and $-6.97 \mathrm{eV}$, respectively (Fig. 3b). These numbers are consistent with previous calculations ${ }^{10,32-36}$. Due to the high instability of the pristine $1 \mathrm{~T}$ phase, the initial binding of hydrogen on a pure 1T-phase basal plane is quite strong, which results in heavy relaxation of the adsorption area. This releases most of the $1 \mathrm{~T}$ energy, making the structure rather inert for the further adsorption of hydrogen atoms $s^{14,37,38}$. Moreover, for the 1 $\mathrm{T}$ phase confined within $2 \mathrm{H}$ phase by interfaces, the strong relaxations at the interfaces have the similar effect as the initial $\mathrm{H}$ adsorption, which prevents the confined $1 \mathrm{~T}$ phase to adsorb more $\mathrm{H}$ atoms favorably (further details in Supplementary Fig. 10). This can explain the absence of $\mathrm{H}$ adsorption at the nanometer-size $1 \mathrm{~T}$ phase as shown in the STM image (Fig. 2d, e). The Gibbs free energy of all the $\mathrm{H}$ adsorption sites in $2 \mathrm{H}-2 \mathrm{H}$ domain boundaries are much smaller than that of the perfect $\mathrm{MoS}_{2}$ basal plane $(1.87 \mathrm{eV})$, indicating that $2 \mathrm{H}-2 \mathrm{H}$ boundaries can indeed break the inertia of the basal plane and enhance the interaction between the $\mathrm{H}$ atom and the adsorption sites. Impressively, the 2H-1T-phase boundaries exhibit $\Delta \mathrm{G}_{\mathrm{H}^{*}}=$ $-0.13 \mathrm{eV}$ (Fig. 3b), very close to that of the Pt (111) surface and Mo-edge of $2 \mathrm{H}-\mathrm{MoS}_{2}$. Thus, phase boundaries in the basal plane of monolayer $\mathrm{MoS}_{2}$ could serve as effective sites to tune hydrogen reaction barriers and optimize the overall kinetics of $\mathrm{H}_{2}$ evolution $^{39,40}$.

Multi-hierarchy monolayer $\mathrm{MoS}_{2}$ catalysts for HER. Based on the above experimental and theoretical results, we can conclude that both domain boundaries and phase boundaries can serve as active sites in HER. More boundaries, in principle, should offer better HER performance. We thus investigated the effect of boundary density in a systematic way. $\mathrm{MoS}_{2}$ electrodes for electrocatalytic HER testing were fabricated from pristine type-II samples (with low density of $2 \mathrm{H}-2 \mathrm{H}$ domain boundaries), pristine type-III samples (with high density of $2 \mathrm{H}-2 \mathrm{H}$ domain boundaries), and a series of heterophase type-III samples with varying density of 2H-1T-phase boundaries. As mentioned above, the heterophase $\mathrm{MoS}_{2}$ can be produced by Ar-plasma bombardments; while the percentage of $1 \mathrm{~T}$-phase in $2 \mathrm{H}$-phase matrix, thus the density of phase boundaries, can be actually tuned by the treatment durations. The illustrated multi-hierarchy 
a

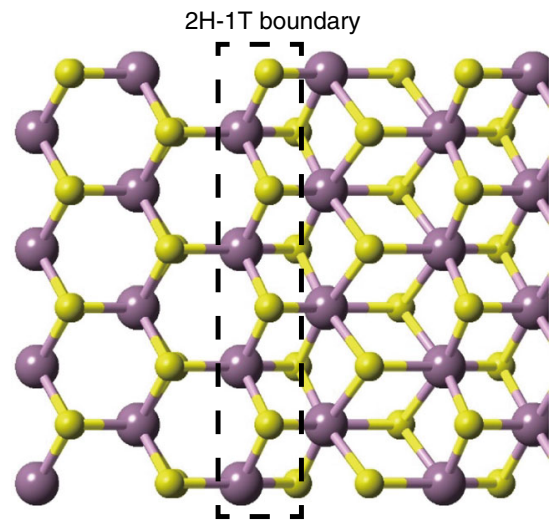

b

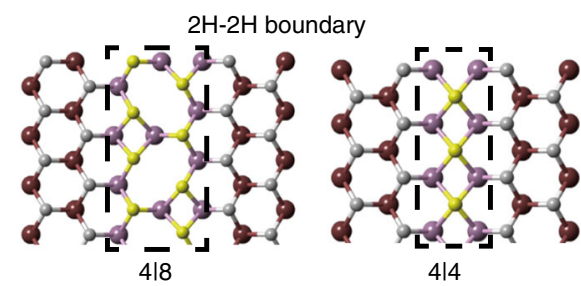

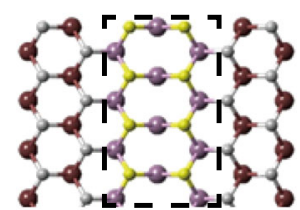

618

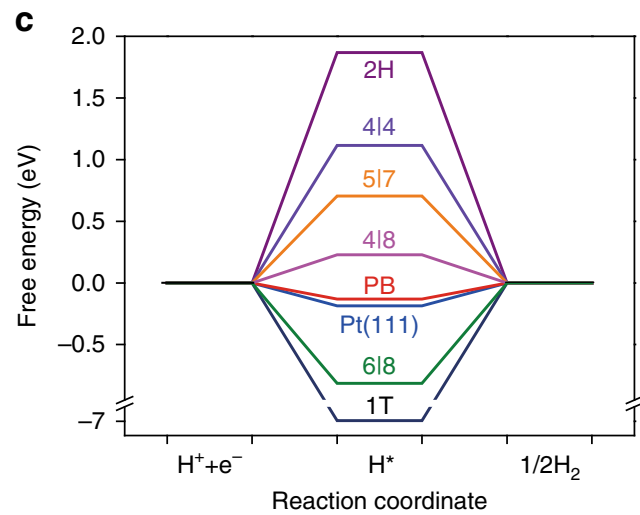

Fig. 3 Theoretical simulations. $\mathbf{a}, \mathbf{b}$ The top views of the atomic model of the zigzag type $2 \mathrm{H}-1 \mathrm{~T}$-phase boundaries and four kinds of $2 \mathrm{H}-2 \mathrm{H}$ boundaries $(4|8,6| 8,4|4,5| 7)$. c A comparison of the Gibbs free energies of the adsorbed $\mathrm{H}$ on $2 \mathrm{H}$-phase of $\mathrm{MoS}_{2}, 1 \mathrm{~T}$-phase of $\mathrm{MoS}_{2}, \mathrm{Pt}(111)$ surface, $2 \mathrm{H}-1 \mathrm{~T}$-phase boundaries ( $\mathrm{PBs}$ ) and four kinds of $2 \mathrm{H}-2 \mathrm{H}$ boundaries in the context of HER

catalysts for HER characterizations is shown in Fig. 4a. ML-MoS catalysts were supported on graphene $(\mathrm{Gr}) / \mathrm{Au}$ films (structure and fabrication details discussed in Supplementary Note 1 and Supplementary Figs. 11-13). Note that the underlying graphene layers, as an internal electron transport channels, can decrease the resistive loss and accelerate electron transport from the $\mathrm{MoS}_{2}$ film to electrodes ${ }^{11}$. Polarization curves (Fig. 4b) and Tafel plots (Fig. 4c) of these samples were measured in $0.5 \mathrm{M}$ sulfuric acid electrolyte using a standard three-electrodes configuration (see the Methods section for more details). Note that, the overpotential at $10 \mathrm{~mA} / \mathrm{cm}^{2}$ decreased linearly with increasing the phase-boundary density of $\mathrm{MoS}_{2}$ tuned by the treatment durations, which confirmed the effect of phase boundaries in HER (further details see Supplementary Figs. 14, 15).

The control sample is a commercial Pt foil, which exhibits a nearly zero overpotential. From these data shown in Fig. $4 \mathrm{~b}$, we can clearly see that samples with either more domain boundaries or more phase boundaries can yield better HER performance in terms of reduced overpotentials and Tafel slopes. The best structure is a composite with both high-density domain and phase boundaries, as shown in the illustrated drawing of Fig. 4a. Notably, the best sample presents a very low onset potential of $\sim 100 \mathrm{mV}$, a low overpotential of $\sim 136 \mathrm{mV}$, a Tafel slope of $\sim 73$ $\mathrm{mV} \mathrm{dec}^{-1}$, an extremely large cathodic current density of $\sim 78$ $\mathrm{mA} \mathrm{cm}^{-2}$ at $\eta=200 \mathrm{mV}$, and an exchange current density of 57 $\mu \mathrm{A} \mathrm{cm} \mathrm{cm}^{-2}$, all of which are better than or comparable to sulfur vacancies (details see Supplementary Fig. 16) and previous results from the edge-dominated $\mathrm{MoS}_{2}$ catalysts ${ }^{33,41,42}$.

A remarkable feature of these composite boundary catalysts is that they can work in both acidic and alkaline conditions with similar performance, considering that only a few candidates could do this job ${ }^{43}$. Polarization curves (Fig. 4d) measured in $1 \mathrm{M} \mathrm{KOH}$ $(\mathrm{pH}=14)$ present an overpotential of $\sim 176 \mathrm{mV}$ at $10 \mathrm{~mA} \mathrm{~cm}^{-2}$, which is slightly larger than that in acidic conditions. We also carried out stability testing, which is another important aspect in practical applications, for the multi-hierarchy $\mathrm{MoS}_{2}$ catalysts in Fig. 4e. Surprisingly, it can work for over $200 \mathrm{~h}$ without any noticeable degradation of current density. We also investigated these two type of boundaries, respectively, and both of them show excellent durability (see Supplementary Fig. 17). Thus we attribute this excellent durability to the stable structures of monolayer $\mathrm{MoS}_{2}$ with $2 \mathrm{H}-2 \mathrm{H}$ and $2 \mathrm{H}-1 \mathrm{~T}$ boundaries. Such structures will not be degraded during the HER process, although they are just monolayers. Finally, the catalysts with composite boundary can also be easily scaled up. As a proof-of-concept demonstration, a 4 -inch wafer-scale catalysts, exhibited in Fig. 4f, was prepared for HER. The wafer-scale catalysts sample still exhibit good HER properties (see Supplementary Fig. 18 and Supplementary Movie 1 for more details).

\section{Discussion}

In conclusion, we have both experimentally and theoretically verified that $2 \mathrm{H}-2 \mathrm{H}$ and $2 \mathrm{H}-1 \mathrm{~T}$ domain boundaries in basal plane of $\mathrm{ML}-\mathrm{MoS}_{2}$ could act as new highly active and tunable catalytic sites for HER. Based on the observed phenomena, we then achieved multi-hierarchy $\mathrm{ML}-\mathrm{MoS}_{2}$ electrocatalysts containing both types of domain boundaries for HER. These electrocatalysts not only show remarkable electrocatalytic performances with a small overpotential of $\sim 0.1 \mathrm{~V}$ and large cathodic currents, but have long-term stability and universality in both acidic and alkaline conditions. Moreover, the ML-MoS electrocatalysts with composite boundaries can be easily scaled up. Our results provide a comprehensive understanding of the HER mechanism for the $\mathrm{MoS}_{2}$ basal plane, as well as a facile route to design high-performance electrocatalysts.

\section{Methods}

Growth of the monolayer $\mathbf{2 H}-\mathrm{MoS}_{\mathbf{2}}$ using CVD method. A three zone furnace was used for CVD growth of $\mathrm{MoS}_{2}$. $\mathrm{SiO}_{2}(300 \mathrm{~nm}) / \mathrm{P}++\mathrm{Si}$ and highly oriented pyrolytic graphite (HOPG) served as substrates. Sulfur (S) (Alfa Aesar 99.9\%) and molybdenum trioxide $\left(\mathrm{MoO}_{3}\right)$ (Alfa Aesar $\left.99.999 \%\right)$ were used as precursors and loaded in zone I and II, respectively. The distance between the two sources was 22 $\mathrm{cm}$. The substrates were put in the third zone. The temperatures of $\mathrm{MoO}_{3}, \mathrm{~S}$, and substrates were 560,120 , and $780^{\circ} \mathrm{C}$, respectively. Each temperature zone was kept stable for $20 \mathrm{~min}$ before the growth. During the growth, argon was used as carrying gas at a flow rate of $130 \mathrm{sccm}$, and the vacuum pressure was kept at 0.67 Torr.

Formation of heterophase $\mathbf{M o S}_{\mathbf{2}}$. Phase transition of $\mathrm{MoS}_{2}$ was also performed in the home-made, remote plasma system reported in our previous work ${ }^{29}$. An 
a

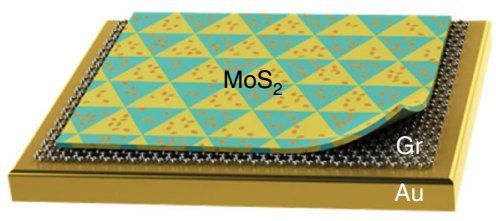

b
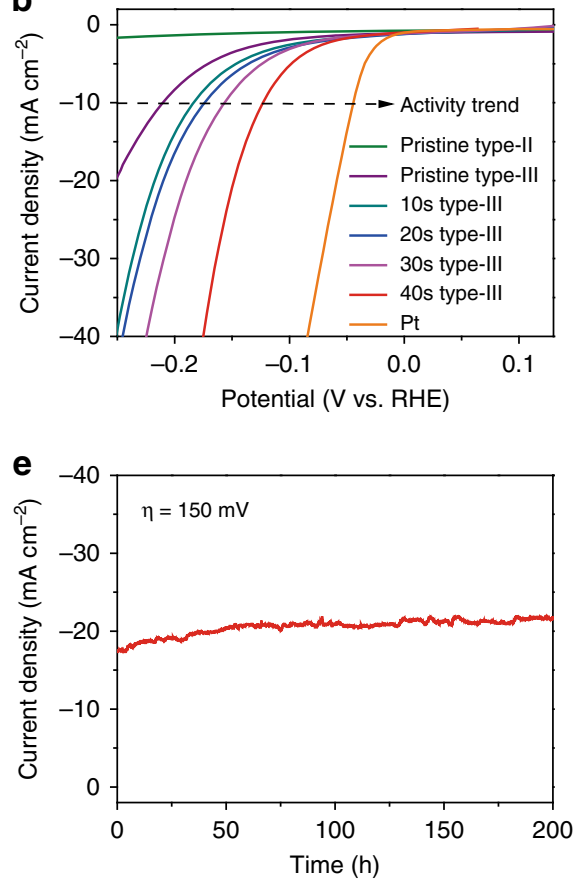

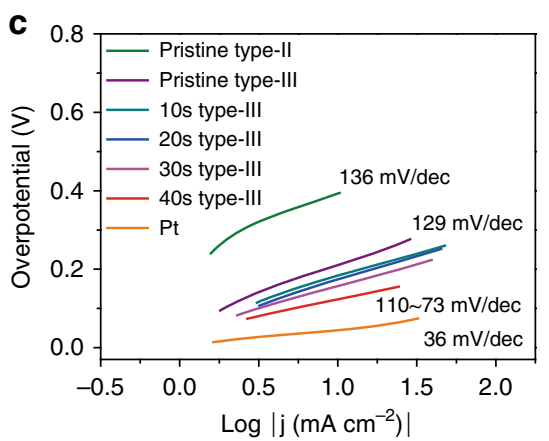

$\mathbf{f}$

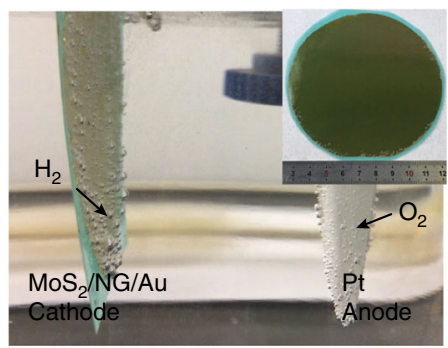

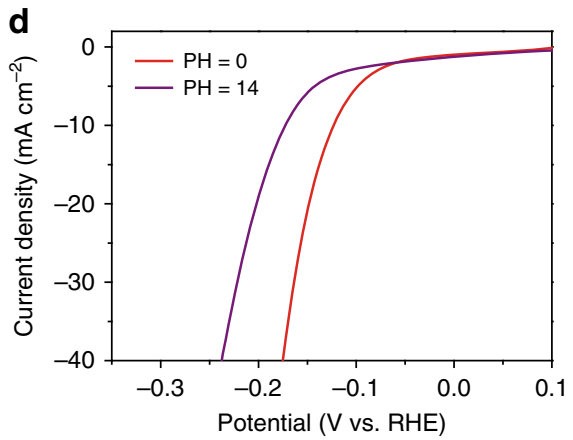

Fig. 4 Multi-hierarchy monolayer $\mathrm{MoS}_{2}$ catalysts for HER. a Schematic structure of the multi-hierarchy $\mathrm{MoS}_{2}$ catalysts with both high density of domain and phase boundaries. b Polarization curves for pristine type-II samples, pristine type-III samples, a series of heterophase type-III samples with different phase boundary densities and Pt. c Tafel plots of the corresponding curves in $\mathbf{b}$. $\mathbf{d} \mathrm{HER}$ performance of a multi-hierarchy $\mathrm{MoS}_{2}$ catalyst in $0.5-\mathrm{M} \mathrm{H} \mathrm{H}_{2} \mathrm{SO}_{4}$ (red curve) and 1-M KOH (purple curve). e Time-dependent current density curve for a multi-hierarchy $\mathrm{MoS}_{2}$ catalyst under static overpotential of $150 \mathrm{mV}$ for $200 \mathrm{~h}$. f Demonstration of the catalytic HER activity in $0.5 \mathrm{M} \mathrm{H}_{2} \mathrm{SO}_{4}$ from a multi-hierarchy $\mathrm{MoS}_{2}$ catalyst with a size of 4 inches in diameter. Inset: photograph of pristine as-grown wafer-scale $\mathrm{MoS}_{2}$ on sapphire substrate

inductively coupled plasma was generated by dispersing a $20 \mathrm{~W}$ RF power at the entrance of a 4 -inch quartz-tube furnace. The pressure in the tube furnace was fixed at $\approx 0.69$ Torr for phase transition by flowing argon at $100 \mathrm{sccm}$ and vacuum pumping. The process was carried out for $10,20,30,40 \mathrm{~s}$ at room temperature, respectively.

Characterization details. The as-grown layer monolayer $\mathrm{MoS}_{2}$ samples were characterized by optical microscopy, AFM (Bruker Icon microscope, tapping mode), Raman spectroscopy (532 nm laser, Horiba Jobin Yvon LabRAM HREvolution Raman), X-ray photoelectron spectroscopy (Kratos Analytical Axis Ultra).

Electrochemical characterizations. All of the electrochemical measurements were performed in a typical three-electrode system on electrochemical workstation (Autolab PGSTAT $302 \mathrm{~N}$ ). A Pt foil or a glassy carbon electrode (for long time test) were used as counter electrodes, and saturated $\mathrm{Ag} / \mathrm{AgCl}$ electrode serve as the reference electrode. The linear sweep voltammetry (LSV) with the scan rate of 5 $\mathrm{mV} \mathrm{s}^{-1}$ was carried out. All the applied potentials were converted to reversible hydrogen electrode (RHE) potentials scaled using the equation $\mathrm{E}$ (vs. RHE) $=\mathrm{E}$ (vs. $\mathrm{Ag} / \mathrm{AgCl})+0.204 \mathrm{~V}+0.0591 \mathrm{VPH}$, after IR correction. The stability tests for the heterophase $\mathrm{MoS}_{2}$ was performed using chronoamperometry at a constant applied overpotential.

Computational details. First-principles calculations based on DFT were carried out by using the Vienna $\mathrm{Ab}$ initio Simulation Package (VASP) ${ }^{7,10}$. The interactions between valence electrons and ions were treated with the projector-augmented wave (PAW) method $^{8}$. The exchange-correlation interactions were described by generalized gradient approximation (GGA) with the Perdew-Burke-Ernzerhof (PBE) functional ${ }^{11}$. The electron wave functions were expanded in a plane-wave basis set with cutoff energy of $520 \mathrm{eV}$. The convergence criterion for residual force on each atom during structure relaxation was set to $0.02 \mathrm{eV} / \AA$, and the geometries were relaxed to minimize the total energy of the system until a precision of $10^{-4} \mathrm{eV}$ was reached.

\section{Data availability}

All data are available from the authors upon reasonable request. All source data underlying Figs. 1a, f, 2c, f, 3c, 4b-e and Supplementary Figs. 1g, h, 3d, e, 5a-c, 7c, 8a, 12a, d, 13a-c, 16, 17 and 18d are provided as a Source Data file.
Received: 26 April 2018 Accepted: 19 February 2019 Published online: 22 March 2019

\section{References}

1. Conway, B. \& Tilak, B. Interfacial processes involving electrocatalytic evolution and oxidation of $\mathrm{H}_{2}$, and the role of chemisorbed $\mathrm{H}$. Electrochim. Acta 47, 3571-3594 (2002).

2. Hinnemann, B. et al. Biomimetic hydrogen evolution: $\mathrm{MoS}_{2}$ nanoparticles as catalyst for hydrogen evolution. J. Am. Chem. Soc. 127, 5308-5309 (2005).

3. Greeley, J., Jaramillo, T. F., Bonde, J., Chorkendorff, I. \& Nørskov, J. K. Computational high-throughput screening of electrocatalytic materials for hydrogen evolution. Nat. Mater. 5, 909 (2006).

4. Chhowalla, M. et al. The chemistry of two-dimensional layered transition metal dichalcogenide nanosheets. Nat. Chem. 5, 263-275 (2013).

5. Cao, X., Tan, C., Zhang, X., Zhao, W. \& Zhang, H. Solution-processed twodimensional metal dichalcogenide-based nanomaterials for energy storage and conversion. Adv. Mater. 28, 6167 (2016).

6. Jaramillo, T. F. et al. Identification of active edge sites for electrochemical $\mathrm{H}_{2}$ evolution from MoS2 nanocatalysts. Science 317, 100-102 (2007).

7. Bonde, J., Moses, P. G., Jaramillo, T. F., Nørskov, J. K. \& Chorkendorff, I. Hydrogen evolution on nano-particulate transition metal sulfides. Faraday Discuss. 140, 219-231 (2009).

8. Merki, D., Vrubel, H., Rovelli, L., Fierro, S. \& Hu, X. Fe, Co, and Ni ions promote the catalytic activity of amorphous molybdenum sulfide films for hydrogen evolution. Chem. Sci. 3, 2515-2525 (2012).

9. Chang, K. et al. $\mathrm{MoS}_{2}$ /graphene cocatalyst for efficient photocatalytic $\mathrm{H}_{2}$ evolution under visible light irradiation. ACS nano 8, 7078-7087 (2014).

10. Xie, J. et al. Controllable disorder engineering in oxygen-incorporated $\mathrm{MoS}_{2}$ ultrathin nanosheets for efficient hydrogen evolution. J. Am. Chem. Soc. 135, 17881-17888 (2013).

11. Bentley, C. L. et al. Electrochemical maps and movies of the hydrogen evolution reaction on natural crystals of molybdenite $\left(\mathrm{MoS}_{2}\right)$ : basal vs. edge plane activity. Chem. Sci. 8, 6583-6593 (2017).

12. Lukowski, M. A. et al. Enhanced hydrogen evolution catalysis from chemically exfoliated metallic $\mathrm{MoS}_{2}$ nanosheets. J. Am. Chem. Soc. 135, 10274-10277 (2013). 
13. Voiry, D. et al. Conducting $\mathrm{MoS}_{2}$ nanosheets as catalysts for hydrogen evolution reaction. Nano. Lett. 13, 6222-6227 (2013).

14. Yin, Y. et al. Contributions of phase, sulfur vacancies, and edges to the hydrogen evolution reaction catalytic activity of porous molybdenum disulfide nanosheets. J. Am. Chem. Soc. 138, 7965 (2016).

15. $\mathrm{Yu}, \mathrm{Y}$. et al. High phase-purity $1 \mathrm{~T}^{\prime}-\mathrm{MoS}_{2}$-and $1 \mathrm{~T}^{\prime}-\mathrm{MoSe}_{2}$-layered crystals Nat. Chem. 10, 638-643 (2018).

16. Voiry, D. et al. The role of electronic coupling between substrate and 2D $\mathrm{MoS}_{2}$ nanosheets in electrocatalytic production of hydrogen. Nat. Mater. 15, 1003 (2016).

17. Li, H. et al. Activating and optimizing $\mathrm{MoS}_{2}$ basal planes for hydrogen evolution through the formation of strained sulphur vacancies. Nat. Mater. 15, 48-53 (2016).

18. Ding, Q., Song, B., Xu, P. \& Jin, S. Efficient electrocatalytic and photoelectrochemical hydrogen generation using $\mathrm{MoS}_{2}$ and related compounds. Chem 1, 699-726 (2016).

19. Zhang, J. et al. Unveiling active sites for the hydrogen evolution reaction on monolayer $\mathrm{MoS}_{2}$. Adv. Mater. 29, 1701955-1701962 (2017).

20. Yin, Y. et al. Synergistic phase and disorder engineering in $1 \mathrm{~T}-\mathrm{MoSe}_{2}$ nanosheets for enhanced hydrogen-evolution reaction. Adv. Mater. 29, 1700311-1700318 (2017).

21. Lin, S.-H. \& Kuo, J.-L. Activating and tuning basal planes of $\mathrm{MoO}_{2}, \mathrm{MoS}_{2}$, and $\mathrm{MoSe}_{2}$ for hydrogen evolution reaction. Phys. Chem. Chem. Phys. 17, 29305-29310 (2015).

22. Lu, A. Y. et al. High-sulfur-vacancy amorphous molybdenum sulfide as a high current electrocatalyst in hydrogen evolution. Small 12, 5530-5537 (2016).

23. $\mathrm{Li}, \mathrm{H}$. et al. Kinetic study of hydrogen evolution reaction over strained $\mathrm{MoS}_{2}$ with sulfur vacancies using scanning electrochemical microscopy. J. Am. Chem. Soc. 138, 5123-5129 (2016).

24. Ouyang, Y. et al. Activating inert basal planes of $\mathrm{MoS}_{2}$ for hydrogen evolution reaction through the formation of different intrinsic defects. Chem. Mater. 28, 4390-4396 (2016).

25. Tsai, C. et al. Electrochemical generation of sulfur vacancies in the basal plane of $\mathrm{MoS}_{2}$ for hydrogen evolution. Nat. Commun. 8, 15113-15120 (2017).

26. Chen, W. et al. Oxygen-assisted chemical vapor deposition growth of large single-crystal and high-quality monolayer $\mathrm{MoS}_{2}$. J. Am. Chem. Soc. 137, 15632-15635 (2015).

27. $\mathrm{Yu}, \mathrm{H}$. et al. Wafer-scale growth and transfer of highly-oriented monolayer $\mathrm{MoS}_{2}$ Continuous films. ACS nano 11, 12001-12007 (2017).

28. Zhang, J. et al. Scalable growth of high-quality polycrystalline $\mathrm{MoS}_{2}$ monolayers on $\mathrm{SiO}_{2}$ with tunable grain sizes. ACS nano 8, 6024-6030 (2014).

29. Zhu, J. et al. Argon plasma induced phase transition in monolayer $\mathrm{MoS}_{2}$. J. Am. Chem. Soc. 139, 10216-10219 (2017).

30. Cho, S. et al. Phase patterning for ohmic homojunction contact in $\mathrm{MoTe}_{2}$. Science 349, 625-628 (2015).

31. Jiao, Y., Zheng, Y., Jaroniec, M. \& Qiao, S. Z. Design of electrocatalysts for oxygen-and hydrogen-involving energy conversion reactions. Chem. Soc. Rev. 44, 2060-2086 (2015).

32. Tauster, S., Pecoraro, T. \& Chianelli, R. Structure and properties of molybdenum sulfide: Correlation of $\mathrm{O}_{2}$ chemisorption with hydrodesulfurization activity. J. Catal. 63, 515-519 (1980).

33. Salmeron, M., Somorjai, G., Wold, A., Chianelli, R. \& Liang, K. The adsorption and binding of thiophene, butene and $\mathrm{H}_{2} \mathrm{~S}$ on the basal plane of MoS2 single crystals. Chem. Phys. Lett. 90, 105-107 (1982).

34. Byskov, L. S., Nørskov, J. K., Clausen, B. S. \& Topsøe, H. DFT calculations of unpromoted and promoted $\mathrm{MoS}_{2}$-based hydrodesulfurization catalysts. J. Catal. 187, 109-122 (1999).

35. Wang, H. et al. Transition-metal doped edge sites in vertically aligned $\mathrm{MoS}_{2}$ catalysts for enhanced hydrogen evolution. Nano. Research 8, 566-575 (2015).

36. Tsai, C., Chan, K., Nørskov, J. K. \& Abild-Pedersen, F. Rational design of $\mathrm{MoS}_{2}$ catalysts: tuning the structure and activity via transition metal doping. Catalysis Science \& Technology 5, 246-253 (2015).

37. Hu, T., Li, R. \& Dong, J. A new $(2 \times 1)$ dimerized structure of monolayer 1Tmolybdenum disulfide, studied from first principles calculations. J. Chem. Phys. 139, 174702-174708 (2013).

38. Tang, Q. \& Jiang, D. Mechanism of hydrogen evolution reaction on $1 \mathrm{~T}-\mathrm{MoS}_{2}$ from first principles. Acs. Catalysis 6, 4953-4961 (2016).
39. Kibsgaard, J., Chen, Z., Reinecke, B. N. \& Jaramillo, T. F. Engineering the surface structure of $\mathrm{MoS}_{2}$ to preferentially expose active edge sites for electrocatalysis. Nat. Mater. 11, 963-969 (2012).

40. Kong, D. et al. Synthesis of $\mathrm{MoS}_{2}$ and $\mathrm{MoSe}_{2}$ films with vertically aligned layers. Nano. Lett. 13, 1341-1347 (2013).

41. Shi, J. et al. Controllable growth and transfer of monolayer $\mathrm{MoS}_{2}$ on Au foils and its potential application in hydrogen evolution reaction. ACS nano $\mathbf{8}$, 10196-10204 (2014).

42. Ye, G. et al. Defects engineered monolayer $\mathrm{MoS}_{2}$ for improved hydrogen evolution reaction. Nano. Lett. 16, 1097-1103 (2016).

43. Yin, J. et al. Ni-C-N nanosheets as catalyst for hydrogen evolution reaction. J. Am. Chem. Soc. 138, 14546-14549 (2016).

\section{Acknowledgements}

G.Z. thanks the supports from NCSF under the grant No. 11834017 and 61888102, the Strategic Priority Research Program of CAS under the grant No. XDB30000000, the Key Research Program of Frontier Science of CAS under the grant No. QYZDB-SSWSLH004, and the National Key R\&D program under grant No. 2016YFA0300904. Y.J. acknowledges support by the National Key R\&D program under Grant No. 2016YFA0300901 and 2017YFA0205003, the National Natural Science Foundation of China under Grant No. 11634001, 11888101 and 21725302, Beijing Municipal Science \& Technology Commision (grant No. Z181100004218006) and the Strategic Priority Research Program of Chinese Academy of Sciences under Grant no. XDB28000000. L.Z is supported by the National Science Foundation of China with Grant No. 11574157. R.Y. acknowledges support by the National Science Foundation of China (NSFC, Grant No. 11574361) and the Youth Innovation Promotion Association CAS (No. 2018013).

\section{Author contributions}

G.Z. and R.Y designed and supervised the research. J.Z., Q.W., Z.W., N.L. and L.D. prepared the samples. J.Z., Q.W. and R.Y. performed AFM, Raman, XPS, and electrochemical characterizations. J.Z., H.Y., J.Z. and W.C. performed TEM characterizations. Z. C.W. and Y.J. performed STM and STS characterizations. H.D. and L.Z. performed calculations. W.W. provided the electrochemical workstation. Data were collected and analyzed by J.Z., Z.C.W., H.D., L.Z., Y.J., D.S. and G.Z. Figures were prepared by J.Z. and M.L. The paper was written by J.Z., L.Z., Y.J. and G.Z. All authors discussed the results and commented on the paper.

\section{Additional information}

Supplementary Information accompanies this paper at https://doi.org/10.1038/s41467 019-09269-9.

Competing interests: The authors declare no competing interests.

Reprints and permission information is available online at http://npg.nature.com/ reprintsandpermissions/

Journal Peer Review Information: Nature Communications thanks the anonymous reviewer(s) for their contribution to the peer review of this work

Publisher's note: Springer Nature remains neutral with regard to jurisdictional claims in published maps and institutional affiliations.

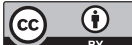

Open Access This article is licensed under a Creative Commons Attribution 4.0 International License, which permits use, sharing adaptation, distribution and reproduction in any medium or format, as long as you give appropriate credit to the original author(s) and the source, provide a link to the Creative Commons license, and indicate if changes were made. The images or other third party material in this article are included in the article's Creative Commons license, unless indicated otherwise in a credit line to the material. If material is not included in the article's Creative Commons license and your intended use is not permitted by statutory regulation or exceeds the permitted use, you will need to obtain permission directly from the copyright holder. To view a copy of this license, visit http://creativecommons.org/ licenses/by/4.0/.

(C) The Author(s) 2019 